\title{
Is a hybrid procedure the gold standard in patients with persistent atrial fibrillation?
}

\section{Dong Seop Jeong ${ }^{1}$, Yoonseo Lee ${ }^{1}$, Young Keun On ${ }^{2}$}

${ }^{1}$ Department of Thoracic and Cardiovascular Surgery, Heart Stroke Vascular Institute, Samsung Medical Center, Sungkyunkwan University School of Medicine, Seoul, Korea

${ }^{2}$ Division of Cardiology, Department of Medicine, Samsung Medical Center, Sungkyunkwan University School of Medicine, Seoul, Korea

Received: April 9, 2020

Revised: May 22, 2020

Accepted: May 26, 2020

Corresponding author:

Dong Seop Jeong

Department of Thoracic and Cardiovascular Surgery, Samsung

Medical Center, Sungkyunkwan University School of Medicine,

81 Irwon-ro, Gangnam-gu, Seoul 06351, Korea

Tel: + 82-2-3410-1278

E-mail:valve@skku.edu

\section{ABSTRACT}

Endocardial radiofrequency catheter ablation is considered to be a useful procedure for paroxysmal atrial fibrillation. However, catheter ablation has difficulties in achieving successful results in patients with persistent or long-standing persistent atrial fibrillation. Currently, the gold standard is surgical ablation (the Cox maze III procedure). However, because surgical ablation requires a sternotomy and extracorporeal cardiopulmonary bypass and has been associated with surgery-related complications, minimally invasive (totally thoracoscopic) and epicardial-endocardial (hybrid) ablation procedures have been developed. This article reviews the primary surgical and hybrid approaches used to treat persistent or long-standing persistent atrial fibrillation.

Keywords: Atrial fibrillation; Catheter ablation; Hybrid; Thoracoscopy
This is an Open Access article distributed under the terms of the Creative Commons Attribution Non-Commercial License (https:// creativecommons.org/licenses/ by-nc/4.0/).

\section{INTRODUCTION}

Atrial fibrillation (AF) is the most prevalent arrhythmia and is of particular clinical concern for its increased morbidity and mortality [1]. In Korea, $5 \%$ of the population older than 65 years and $10 \%$ of the population older than 80 years have a history of AF. Although the rapid evolution of catheter ablation has led to successful results in treating paroxysmal AF, persistent and long-standing persistent AF remain major clinical challenges with unfavorable outcomes. Meta-analyses on catheter ablation have demonstrated that $57 \%$ of patients maintained normal sinus rhythm without an antiarrhythmic drug after a single procedure, and $71 \%$ of patients achieved a normal sinus rhythm after two or more interventions [2].

Surgical treatment (i.e., maze procedure) has obvious limitations because of its technical difficulty, invasiveness, and requirement for cardiopulmonary bypass. Currently, surgical procedures for AF have shifted from full sternotomy and the cut-and-sew maze operation to minimally invasive techniques using a thoracoscopic approach, which makes it possible to perform the procedure without cardiopulmonary bypass. With this development, the thoracoscopic approach as a single procedure is safer and carries higher rates of arrhythmia-free survival than catheter ablations [3]. However, limitations still exist because of the characteristics of tho- 
racoscopic procedures. Contiguous and transmural lesions are not always guaranteed with this approach [4] and it is particularly difficult to ablate the mitral isthmus since this procedure approaches from the epicardial surface [5].

\section{CATHETER ABLATION}

AF results from electrophysiological disturbances within the atria. Electrical activities from multiple sources, especially those from pulmonary veins, disrupt normal sinus rhythm. Since Haissaguerre et al. [6] reported that ectopic beats originating from pulmonary veins are important culprits of AF, these sites have been the key target of catheter ablation. Although circumferential isolation of both pulmonary veins is adequate for maintaining normal sinus rhythm in patients with paroxysmal AF [7], patients with persistent and long -standing persistent AF have high rates of recurrence [8].

Non-pulmonary vein triggers are also important in the initiation of AF and are common targets of ablation during pulmonary vein isolation. Paroxysmal and persistent forms of AF are not free from these triggers, including the left atrial appendage, posterior wall of the left atrium, superior vena cava, coronary sinus, ligament of Marshall, and the mitral isthmus. However, at most, $45 \%$ of patients with persistent or long-standing persistent AF were successfully freed from AF after a single catheter ablation, and $65 \%$ after multiple ablations. Even worse records have been reported in longterm results of catheter ablation in patients with persistent AF, with a 20\% success rate after 1 procedure at 5 years and an approximately $45 \%$ success rate after multiple procedures at 5 years [9]. It is important to note that these values are derived from research in which catheter ablation was per- formed in patients with drug-refractory AF. Nevertheless, the low success rate in patients with persistent $A F$ has resulted in strategies that aim to improve ablation and surgical interventions with an acceptable risk-benefit profile.

\section{CONVENTIONAL ARRHYTHMIA SURGERY}

First introduced in 1987, the initial surgical Cox maze procedure was composed of complicated "maze" incisions in both atria with the aim of interrupting macro-reentrant circuits as well as isolating the sinus node and interrupting Bachmann's bundle [10-12]. The Cox maze procedure shed new light on a non-pharmacological strategy of AF treatment and was developed into the Cox maze III procedure. In an effort to overcome injury to Bachmann's bundle, the entire left atrial dome incision was moved posterior to the superior vena cava. With atrial septostomy posterior to the superior vena cava orifice, the left atrium is exposed more simply with less effort, and pericardial patching for superior vena cava repair is no longer necessary. This much easier procedure solves both the chronotropic incompetence of the sinus node and the occasional dysfunction of the left atrium. Lesion sets of the Cox maze III procedure have become the standard over the past 30 years. Prasad et al. [13] reported that $96.6 \%$ of patients were free from symptomatic AF at 5.4 years, with no difference observed between those who underwent the Cox maze III procedure alone and those who had concomitant cardiac operations. Gomes et al. [14] recently reported rates of sinus rhythm maintenance after the Cox maze III procedure of $88 \%, 85.1 \%$, and $80.6 \%$ at 6,24 , and 36 months, respectively.

The Cox maze IV procedure has been developed as a result of the technical difficulties of the cut-and-sew Cox maze III

Table 1. Arrhythmia outcomes over 2 years

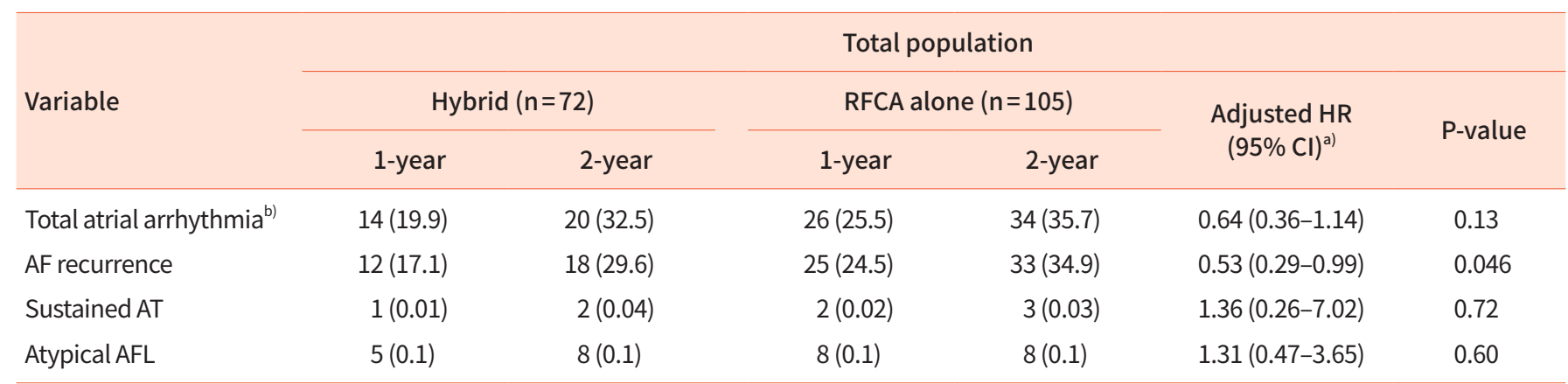

Values are presented as number (\%). The percentages shown are Kaplan-Meier estimates form the intention to treat analysis. Significant P-value is $<0.05$. The $H R$ is for the hybrid group as compared with the RFCA alone group.

$\mathrm{RFCA}$, radiofrequency catheter ablation; $\mathrm{HR}$, hazard ratio; $\mathrm{Cl}$, confidence interval; $\mathrm{AF}$, atrial fibrillation; $\mathrm{AT}$, atrial tachycardia; $\mathrm{AFL}$, atrial flutter. ${ }^{a}$ Relevant covariates considered for analysis were age, body mass index, male, types of AF, LA volume index, and antiarrhythmic drugs prescribed

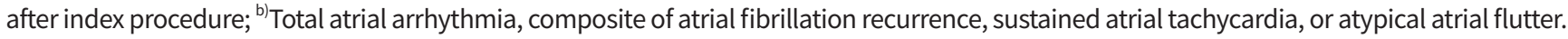


procedure, better understanding of the electrophysiological anatomy of AF, and the advent of various energy sources for ablation. In this new procedure, ablation lines are created rather than atrial incisions, which has been as effective as the Cox maze III procedure $[15,16]$. This result contributed to the development of minimally invasive surgery and is characterized by its epicardial procedure and the lack of a need for cardiopulmonary bypass.

\section{HYBRID PROCEDURE}

The recent hybrid approach is an attractive alternative that overcomes the limitations of epicardial and endocardial ablations. Current data have elucidated the necessity for endocardial electrophysiological confirmation. As surgical lesions alone were shown to have difficulties in achieving complete isolation, staged catheter ablation after the window period was required $[17,18]$. Even though hybrid techniques need a multidisciplinary approach, which is time-consuming and challenging in terms of coordination and equipment, collaboration between surgeons and electrophysiologists should be encouraged for better rhythm outcomes of long-standing AF. The hybrid procedure in our institution consists of com- plete thoracoscopic ablation and postprocedural electrophysiological confirmation at 3 months postoperatively.

\section{Minimally invasive totally thoracoscopic ablation}

In our institution, "totally thoracoscopic" was defined as a video-assisted thoracoscopic surgical technique without the aid of mini-thoracotomy, the da Vinci system (Intuitive Surgical, Sunnyvale, CA, USA), or cardiopulmonary bypass. This approach required only three holes: two 5-mm in size and one $10-\mathrm{mm}$ in size. The procedure starts at the right side with a 5-mm port introduced in the fourth intercostal space at the midaxillary line. Then, to expand the operative field and depress the diaphragm, carbon dioxide insufflation was done followed by placing the remaining two ports in the third intercostal space at the anterior axillary line and the sixth intercostal space at the midaxillary line, respectively. An AtriCure Isolator Transpolar Clamp (AtriCure, Cincinnati, OH, USA) was positioned around the pulmonary vein antrum, and clamping was performed six times using bipolar radiofrequency energy to isolate the pulmonary vein (Fig. 1A, B). Additionally, a linear pen device (AtriCure) (Fig. 1C) is used to create epicardial superior and inferior ablation lines connecting both pulmonary vein isolation lines. Subsequently, an
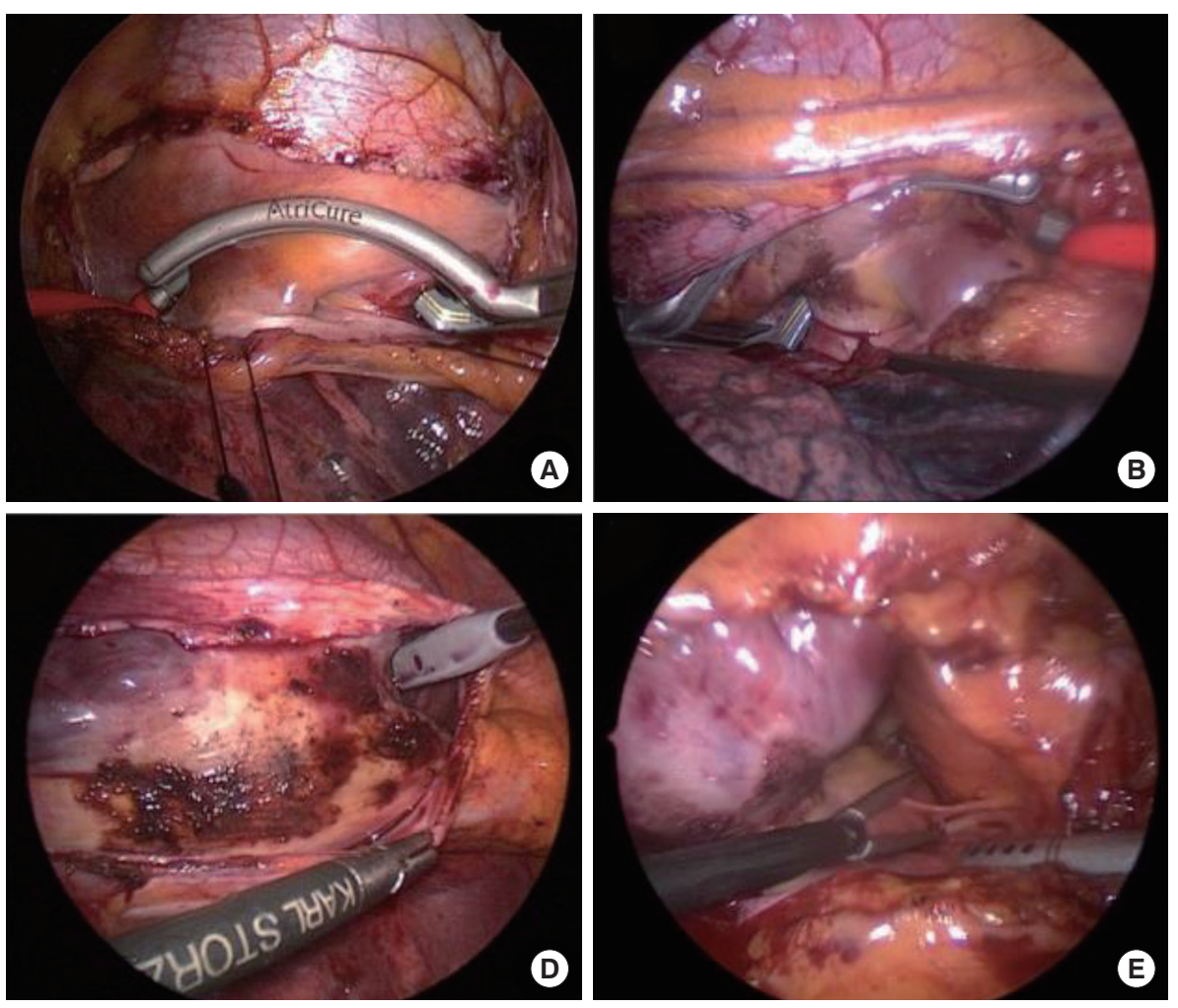
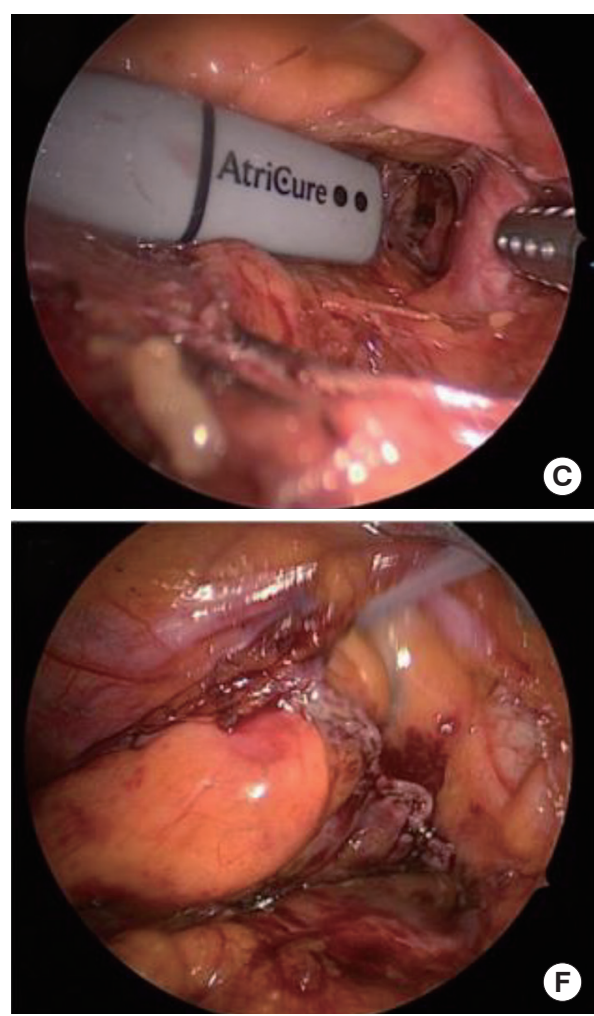

Fig. 1. Operative photographs of thoracoscopic ablation. (A) Right pulmonary vein isolation. (B) Left pulmonary vein isolation. (C) Left atrial roof linear ablation. (D) Ganglionated plexus ablation. (E) Division of ligament of Marshall vein. (F) After resection of left atrial appendage. 
ablation of ganglionated plexuses with bipolar radiofrequency energy with the aid of high-frequency stimulation is performed (Fig. 1D). Ablation lines were confirmed by pace testing using the AtriCure Cooltip pen. The procedure was then repeated on the left side. The ligament of Marshall was dissected and ablated before pulmonary vein and ganglionated plexus ablation (Fig. 1E). After the completion of all ablations and confirmation of conduction block, the left atrial auricle was obliterated with an endoscopic stapling device (Fig. 1F).

All patients underwent 24-hour Holter monitoring at 2 weeks, 3 months, 6 months, and 1 year. For those found to have more than $5 \%$ AF burden during 24-hour Holter monitoring, 2-week monitoring was performed to confirm AF recurrence. If necessary, electric or chemical cardioversion is used to recover sinus rhythm during follow-up within the 3-month blanking period. With the exception of some cases decided by cardiologists considering patients' previous stroke history and/or CHA2DS-VASc score, anticoagulation with warfarin was stopped at 3 months postoperatively.

\section{Endocardial electrophysiological procedure}

Electrophysiological studies were performed during follow-up (3 months after totally thoracoscopic ablation) in the hybrid group. Electrodes were inserted into the left femoral vein percutaneously, and one 6 -F quadripolar electrode catheter was located in the right ventricular apex using fluoroscopic guidance and one 7-F duo-decapolar catheter was located at the crista terminalis of the right atrium and coronary sinus. After positioning the catheters, two SL1 sheaths (St. Jude Medical, St. Paul, MN, USA) were inserted into the left atrium through transseptal access using fluoroscopy and blood pressure monitoring. Right after sheath insertion, intravenous heparin for systemic anticoagulation was initiated, checking the activated clotting time every 30 minutes to achieve a target range from 300 to 350 seconds. A multiview of the pulmonary venogram was obtained using a standard transseptal sheath. The Carto 3 system (Biosense Webster, Diamond Bar, CA, USA) is used to map the left atrial geometry and confirm the location of the catheters. One Lasso NAV catheter (Biosense Webster) with a diameter of 15 to $25 \mathrm{~mm}$ was inserted into the pulmonary vein, and its potential was checked. In patients who required three-dimensional mapping, a Lasso catheter and a Thermocool ablation catheter (Biosense Webster) were placed in the left atrium using single or double transseptal access. Ablation strategies were devised to remove residual potentials in the four pulmonary vein antra. In patients with a history of long-standing AF or with perioperative events of atrial flutter, cavotricuspid isthmus ablation was performed. If sinus rhythm conversion was not achieved after removing residual potentials around the pulmonary vein antra, ablation of the mitral isthmus, mitral annulus, roof line, septal line, and antrum of the superior vena cava was done.

\section{CURRENT OUTCOMES}

\section{Safety of totally thoracoscopic ablation}

We experienced two major bleeding episodes during the learning period [19]. After that, until 2018, no additional bleeding events occurred in 296 cases. We thought that major bleeding could be overcome after a learning curve of 50 patients. Phrenic nerve injury was also a concern since it can cause respiratory complications. Phrenic nerve injury was caused by heat using unipolar electrocautery. Fortunately, we were able to dramatically reduce the prevalence of phrenic nerve palsy with the use of bipolar-type electrocautery (harmonic scalpel). Abugattas et al. [20] reported a rate of early complications of $17 \%$ in their study, but most complications were minor and patients recovered quickly without any sequelae. The only remarkable complication was pericarditis. We prescribe colchicine to all cases after thoracoscopic ablations to reduce the risk of acute pericardial reactions. Nevertheless, $3 \%$ of patients in our cohorts complained of chest pain caused by pericarditis and required readmission.

\section{Rhythm outcomes}

Earlier studies described the results of hybrid AF ablation in more than 50 patients. Pison et al. [21] reported a $74 \%$ success rate in 78 patients, and Gehi et al. [22] reported a $66 \%$ success rate in 101 patients, including patients receiving antiarrhythmic therapy. Recently, Bulava et al. [23] demonstrated that among 70 patients with persistent $A F$, treatment with hybrid epicardial and endocardial radiofrequency ablation resulted in $77 \%$ of patients with arrhythmia-free survival and off antiarrhythmic drugs after 12 months. However, the use of previously ineffective antiarrhythmic drugs and redo-ablation procedures increased the arrhythmia-free survival rate to $97 \%$ [23]. The results of the current study are in accordance with our experiences in our institution. Among the 172 patients undergoing thoracoscopic ablation in our institution with a median follow-up duration of 1 year (Table 1) [24,25], patients suffered recurrent significant atrial tachyarrhythmia [24]. Additional catheter ablation was done on 22 of 24 patients and sinus rhythm was restored in 17 patients (Fig. 2). In 


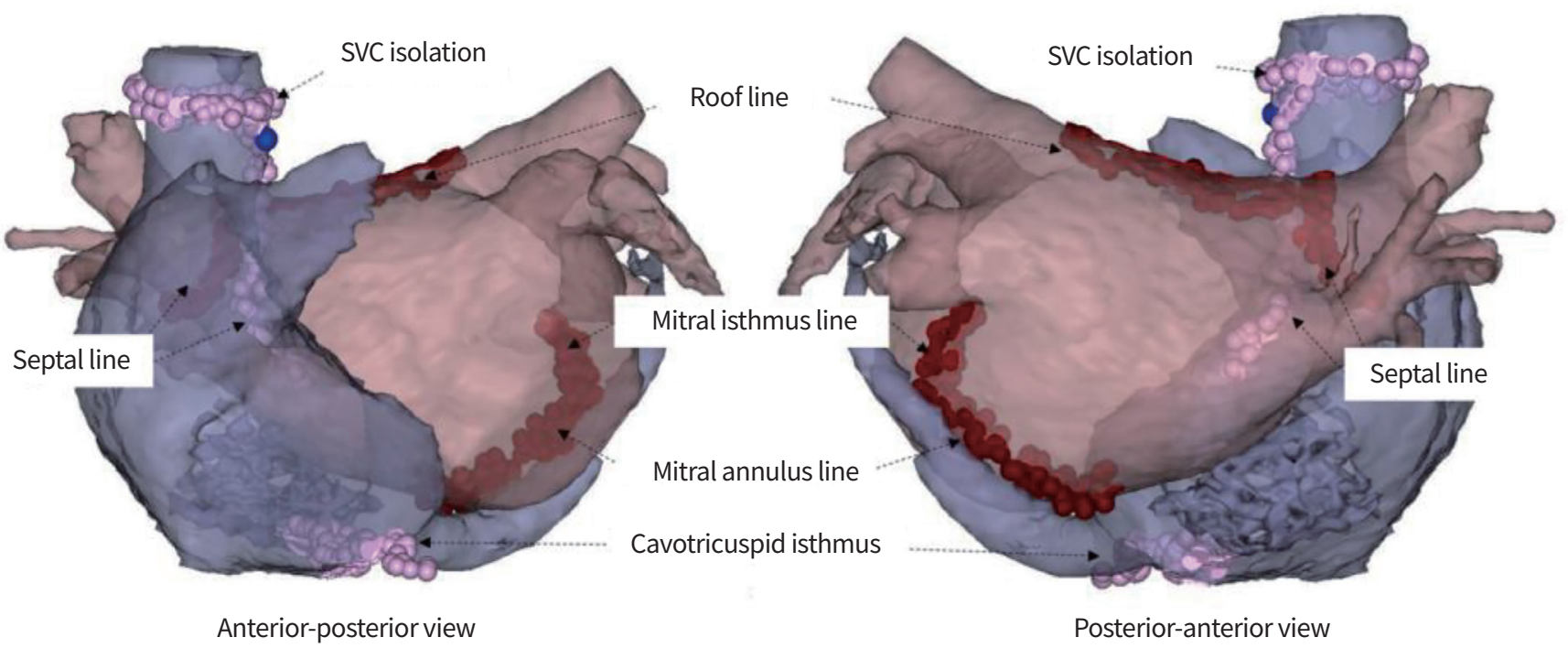

Fig. 2. Additional ablation lesions after thoracoscopic ablation. Endocardial mitral isthmus and septal line ablation are impossible thoracoscopically. SVC, superior vena cava.
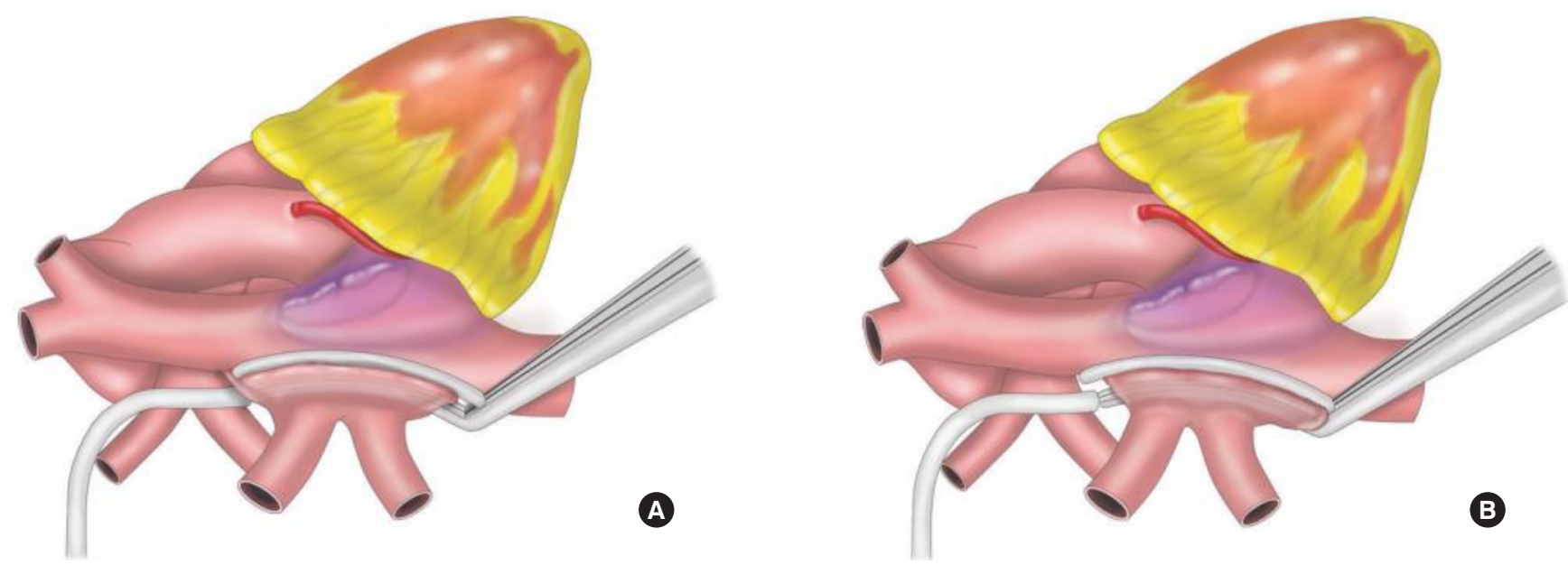

Fig. 3. Inadequate pulmonary vein isolation using bipolar clamp in highly dilated left atrium. (A) Gap in the superior side of the right upper pulmonary vein. (B) Gap in the inferior side of the right lower pulmonary vein.

summary, 165 patients (96\%) showed normal sinus rhythm after hybrid ablation during follow-up, although the 1-year success rate of epicardial thoracoscopic ablation was $86 \%$ (148/172). Based on this clinical evidence, we agree that in the case of persistent AF, the hybrid approach seems to be very promising.

Reconnection is considered to be one of the most important factors of recurrent atrial tachyarrhythmia after surgical ablation of AF $[26,27]$. Our study demonstrated that because insufficient energy is transmitted at the end of the jaws, the superior or inferior ridge of the pulmonary veins is commonly involved in the reconnection sites after surgical ablation (Fig. 3) [19]. According to our previously mentioned recent experience [24,25], pulmonary vein gaps were detected in 12 of 24 patients who showed recurrent symptomatic atrial tachyarrhythmia after epicardial thoracoscopic ablation (Fig. 4). Even with confirmation of pulmonary vein isolation by the intraoperative exit block, residual gaps were observed. To reduce atrial tachyarrhythmia, we added lesions between the superior vena cava and inferior vena cava using a cryoprobe. A circular lesion in the superior vena cava using a bipolar clamp was made when a significantly enlarged right atrium was observed by thoracoscopy.

Timing of endocardial electrophysiological confirmation There remains controversy over the proper timing of post- 


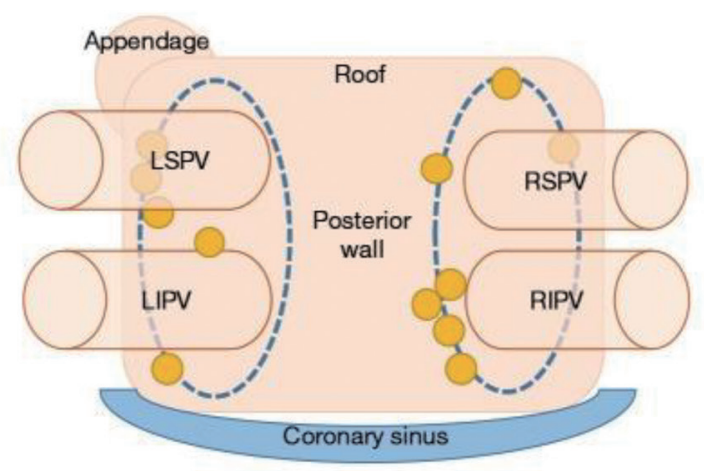

\begin{tabular}{lcccc}
\hline & Anterior ridge & Carina & Roof & Posterior ridge \\
\hline Left PV $(n=5)$ & $4(80 \%)$ & $1(20 \%)$ & 0 & 0 \\
Right PV $(n=7)$ & $1(14.3 \%)$ & 0 & $1(14.3 \%)$ & $5(71.4 \%)$ \\
\hline
\end{tabular}

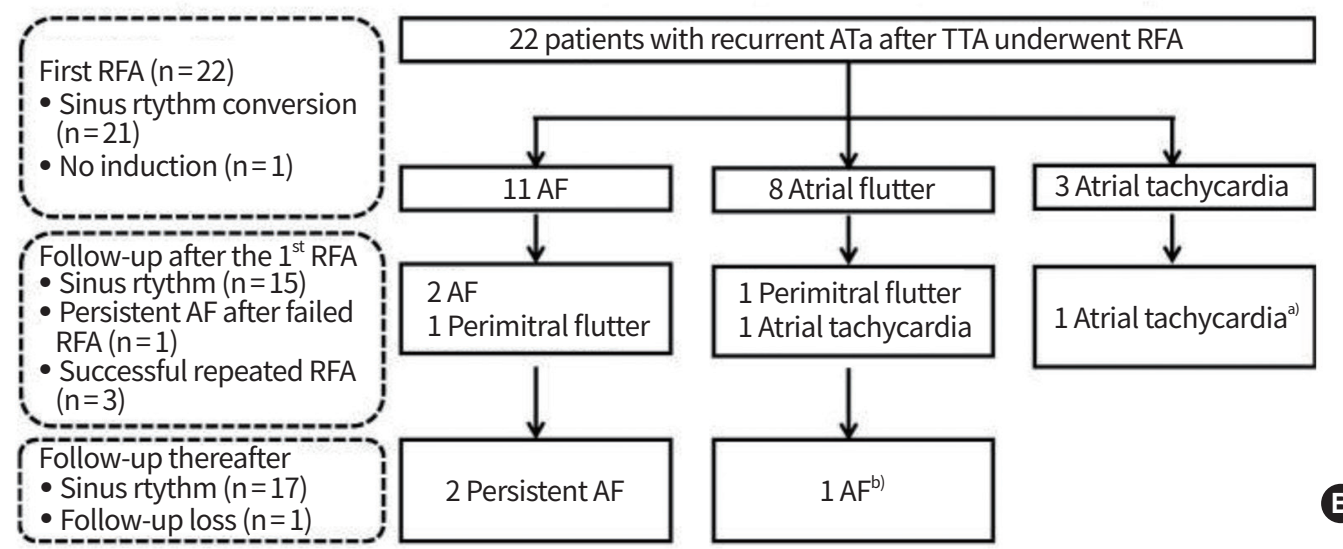

Fig. 4. Outcomes of postoperative electrophysiology studies and radiofrequency catheter ablations. (A) Pulmonary vein gaps. (B) Rhythm outcomes after additional catheter ablations. LSPV, left superior pulmonary vein; RSPV, right superior pulmonary vein; LIPV, left inferior pulmonary vein; RIPV, right inferior pulmonary vein; PV, pulmonary vein; RFA, radiofrequency catheter ablation; ATa, atrial tachyarrhythmia; TTA, totally thoracoscopic ablation; AF, atrial fibrillation. ${ }^{\text {a) }}$ On amiodarone; ${ }^{\text {b) }}$ Asymptomatic.

procedural endocardial ablation, which is done for generating additional linear lines for complete pulmonary vein isolation as it is crucial to make transmural ablation lines for AF treatment [28]. In our center, a simultaneous hybrid strategy was not adopted for certain reasons, including a lack of hybrid facilities and a high risk of significant bleeding during the hybrid procedure. Although a simultaneous strategy has benefits in theory, there are limitations in conforming pulmonary vein isolation immediately after epicardial ablation. Magnano et al. [29] reported that a lesion which appears completely transmural might turn into an incomplete lesion over time because of the edematous condition of tissues immediately post-operation. In addition, Bulava et al. [30] showed that pulmonary vein lesions made immediately after epicardial ablation are not directly associated with atrial ar- rhythmias over time. In our early experience, we performed endocardial electrophysiological confirmations 5 days after surgery and found an absence of AF in $93 \%$ of patients at 2 years [19]. To prove the efficacy of early endocardial electrophysiological confirmation, we conducted a randomized controlled prospective study [31]. In that trial, $89 \%$ of patients (40/45) showed normal sinus rhythm 1 year postoperatively, and additional catheter ablations were done on five patients ( $23 \%, 5 / 22$, the hybrid group) because residual potential was detected in the left atrium. At 12 months, normal sinus rhythm was achieved without additional intervention in $70 \%$ of patients undergoing thoracoscopic ablation. Hence, the timing of the endocardial procedure was changed from 3 to 5 days after the operation in all patients to 3 months postoperatively (window period) only in patients showing recur- 
rent atrial arrhythmias, which is in line with a previously published report [20].

\section{CONCLUSION}

Electrophysiological confirmation following thoracoscopic ablation might be helpful in improving rhythm outcomes and decreasing the prevalence of late atrial tachyarrhythmia. We suggest that electrophysiological back-up should be considered for certain patients with atrial arrhythmia that is refractory to thoracoscopic ablation, and this is a more reasonable approach than a simultaneous or staged hybrid approach for all patients considering cost-effectiveness.

\section{CONFLICTS OF INTEREST}

No potential conflict of interest relevant to this article was reported.

\section{ORCID}

Dong Seop Jeong https://orcid.org/0000-0002-6947-8403

Yoonseo Lee https://orcid.org/0000-0003-4197-4728

Young Keun On https://orcid.org/0000-0003-1025-7283

\section{AUTHOR CONTRIBUTIONS}

Conception or design: YKO.

Acquisition, analysis, or interpretation of data: DSJ.

Drafting the work or revising: YL.

Final approval of the manuscript: DSJ.

\section{REFERENCES}

1. Ahlsson A, Fengsrud E, Bodin L, Englund A. Postoperative atrial fibrillation in patients undergoing aortocoronary bypass surgery carries an eightfold risk of future atrial fibrillation and a doubled cardiovascular mortality. Eur J Cardiothorac Surg 2010;37:1353-9.

2. Noheria A, Kumar A, Wylie JV Jr, Josephson ME. Catheter ablation vs antiarrhythmic drug therapy for atrial fibrillation: a systematic review. Arch Intern Med 2008;168:581-6.

3. Boersma LV, Castella M, van Boven W, Berruezo A, Yilmaz A, Nadal M, et al. Atrial fibrillation catheter ablation versus surgical ablation treatment (FAST): a 2-center randomized clinical trial. Circulation 2012;125:23-30.

4. Pison L, La Meir M, van Opstal J, Blaauw Y, Maessen J, Cri- jns HJ. Hybrid thoracoscopic surgical and transvenous catheter ablation of atrial fibrillation. Am Coll Cardiol 2012;60:54-61.

5. Kurfirst V, Mokracek A, Bulava A, Canadyova J, Hanis J, Pesl L. Two-staged hybrid treatment of persistent atrial fibrillation: short-term single-centre results. Interact Cardiovasc Thorac Surg 2014;18:451-6.

6. Haissaguerre M, Jais P, Shah DC, Takahashi A, Hocini M, Quiniou G, et al. Spontaneous initiation of atrial fibrillation by ectopic beats originating in the pulmonary veins. N Engl J Med 1998;339:659-66.

7. January CT, Wann LS, Alpert JS, Calkins H, Cigarroa JE, Cleveland JC Jr, et al. 2014 AHA/ACC/HRS guideline for the management of patients with atrial fibrillation: a report of the American College of Cardiology/American Heart Association Task Force on practice guidelines and the Heart Rhythm Society. Circulation 2014;130:e199267.

8. Brooks AG, Stiles MK, Laborderie J, Lau DH, Kuklik P, Shipp NJ, et al. Outcomes of long-standing persistent atrial fibrillation ablation: a systematic review. Heart Rhythm 2010;7:835-46.

9. Tilz RR, Rillig A, Thum AM, Arya A, Wohlmuth P, Metzner A, et al. Catheter ablation of long-standing persistent atrial fibrillation: 5-year outcomes of the Hamburg Sequential Ablation Strategy. J Am Coll Cardiol 2012;60:1921-9.

10. Cox JL. The surgical treatment of atrial fibrillation. IV. Surgical technique. J Thorac Cardiovasc Surg 1991;101: 584-92.

11. Cox JL, Boineau JP, Schuessler RB, Ferguson TB Jr, Cain $M E$, Lindsay $B D$, et al. Successful surgical treatment of atrial fibrillation. Review and clinical update. JAMA 1991; 266:1976-80.

12. Damiano RJ Jr, Gaynor SL, Bailey M, Prasad S, Cox JL, Boineau JP, et al. The long-term outcome of patients with coronary disease and atrial fibrillation undergoing the Cox maze procedure. J Thorac Cardiovasc Surg 2003; 126:2016-21.

13. Prasad SM, Maniar HS, Camillo CJ, Schuessler RB, Boineau JP, Sundt TM 3rd, et al. The Cox maze III procedure for atrial fibrillation: long-term efficacy in patients undergoing lone versus concomitant procedures. J Thorac Cardiovasc Surg 2003;126:1822-8.

14. Gomes GG, Gali WL, Sarabanda AVL, Cunha CRD, Kessler IM, Atik FA. Late results of cox maze III procedure in patients with atrial fibrillation associated with structural heart disease. Arq Bras Cardiol 2017;109:14-22. 


\section{PRECISION AND FUTURE MIEDICINE}

Effectiveness of a hybrid procedure

15. Lawrance CP, Henn MC, Miller JR, Sinn LA, Schuessler RB, Maniar HS, et al. A minimally invasive Cox maze IV procedure is as effective as sternotomy while decreasing major morbidity and hospital stay. J Thorac Cardiovasc Surg 2014;148:955-61.

16. Damiano RJ Jr. Alternative energy sources for atrial ablation: judging the new technology. Ann Thorac Surg 2003; 75:329-30.

17. Beyer E, Lee R, Lam BK. Point: minimally invasive bipolar radiofrequency ablation of lone atrial fibrillation: early multicenter results. J Thorac Cardiovasc Surg 2009;137: 521-6.

18. Holmes DR, Reddy VY, Turi ZG, Doshi SK, Sievert H, Buchbinder $\mathrm{M}$, et al. Percutaneous closure of the left atrial appendage versus warfarin therapy for prevention of stroke in patients with atrial fibrillation: a randomised non-inferiority trial. Lancet 2009;374:534-42.

19. On YK, Park KM, Jeong DS, Park PW, Lee YT, Park SJ, et al. Electrophysiologic results after thoracoscopic ablation for chronic atrial fibrillation. Ann Thorac Surg 2015;100: 1595-602.

20. Abugattas JP, lacopino S, Moran D, De Regibus V, Takarada K, Mugnai G, et al. Efficacy and safety of the second generation cryoballoon ablation for the treatment of paroxysmal atrial fibrillation in patients over 75 years: a comparison with a younger cohort. Europace 2017;19: 1798-803.

21. Pison L, Gelsomino S, Luca F, Parise O, Maessen JG, Crijns $\mathrm{HJ}$, et al. Effectiveness and safety of simultaneous hybrid thoracoscopic and endocardial catheter ablation of lone atrial fibrillation. Ann Cardiothorac Surg 2014;3:38-44.

22. Gehi AK, Mounsey JP, Pursell I, Landers M, Boyce K, Chung EH, et al. Hybrid epicardial-endocardial ablation using a pericardioscopic technique for the treatment of atrial fibrillation. Heart Rhythm 2013;10:22-8.
23. Bulava A, Mokracek A, Hanis J, Eisenberger M, Kurfirst V, Dusek L. Correlates of arrhythmia recurrence after hybrid epi- and endocardial radiofrequency ablation for persistent atrial fibrillation. Circ Arrhythm Electrophysiol 2017;10:e005273.

24. Gwag HB, Jeong DS, Hwang JK, Park SJ, On YK, Kim JS, et al. Characteristics of symptomatic recurrent tachyarrhythmia after thoracoscopic ablation for persistent atrial fibrillation. Pacing Clin Electrophysiol 2019;42:686-93.

25. Hwang JK, Jeong DS, Gwag HB, Park KM, Ahn J, Carriere K, et al. Staged hybrid procedure versus radiofrequency catheter ablation in the treatment of atrial fibrillation. PLoS One 2018;13:e0205431.

26. Wazni OM, Saliba W, Fahmy T, Lakkireddy D, Thal S, Kanj M, et al. Atrial arrhythmias after surgical maze: findings during catheter ablation. J Am Coll Cardiol 2006;48:1405-9.

27. Zeng Y, Cui Y, Li Y, Liu X, Xu C, Han J, et al. Recurrent atrial arrhythmia after minimally invasive pulmonary vein isolation for atrial fibrillation. Ann Thorac Surg 2010;90:510-5.

28. Calkins H, Hindricks G, Cappato R, Kim YH, Saad EB, Aguinaga L, et al. 2017 HRS/EHRA/ECAS/APHRS/SOLAECE expert consensus statement on catheter and surgical ablation of atrial fibrillation. Heart Rhythm 2017;14:e275444.

29. Magnano AR, Argenziano M, Dizon JM, Vigilance D, Williams M, Yegen $\mathrm{H}$, et al. Mechanisms of atrial tachyarrhythmias following surgical atrial fibrillation ablation. J Cardiovasc Electrophysiol 2006;17:366-73.

30. Bulava A, Mokracek A, Hanis J, Kurfirst V, Eisenberger M, Pesl L. Sequential hybrid procedure for persistent atrial fibrillation. J Am Heart Assoc 2015;4:e001754.

31. Choi MS, On YK, Jeong DS, Park KM, Park SJ, Kim JS, et al. Usefulness of postprocedural electrophysiological confirmation upon totally thoracoscopic ablation in persistent atrial fibrillation. Am J Cardiol 2020;125:1054-62. 